\title{
Asymptotically Holomorphic Embeddings of Contact Manifolds in Projective Spaces
}

\author{
Vicente Muñoz, Fran Presas, and Ignacio Sols
}

\begin{abstract}
Using asymptotically holomorphic techniques for contact manifolds, we construct embeddings of a $(2 n-1)$-dimensional cooriented contact manifold $(C, \theta)$ into the projective space $\mathbb{C} P^{2 n+1}$ which are weakly dominate.
\end{abstract}

In [MPS] the authors produce symplectic embeddings of a $2 n$-symplectic manifold $(M, \omega)$ with $[\omega] / 2 \pi$ an integer cohomology class into the projective space $\mathbb{C} P^{2 n+1}$, such that the pull-back of the Fubini-Study symplectic form is cohomologous to $k \omega$ for $k$ large. The particularity of these newly constructed embeddings is that they are almost holomorphic in the following sense: let $J$ be an $\omega$-compatible almost complex structure on $M$ and fix an hermitian line bundle $L \rightarrow M$ with $c_{1}(L)=[\omega] / 2 \pi$, and a connection on $L$ with curvature $-i \omega$. Then we find sequences of sections $s_{k}=\left(s_{k}^{0}, s_{k}^{1}, \ldots, s_{k}^{2 n+1}\right)$ of the bundles $\mathbb{C}^{2 n+2} \otimes L^{\otimes k}$ such that

(1) $s_{k}$ are asymptotically holomorphic, i.e., $\left|\nabla^{p} s_{k}\right|=O(1)$ and $\left|\nabla^{p-1} \bar{\partial} s_{k}\right|=$ $O\left(k^{-1 / 2}\right)$, for all $p \geq 1$.

(2) $s_{k}$ are projectizable, i.e., there is an uniform $\gamma>0$ (independent of $k$ ) such that $\left|s_{k}\right| \geq \gamma$ on the whole of $M$. Then we have defined the maps $\phi_{k}=\mathbb{P}\left(s_{k}\right)=\left[s_{k}^{0}, \ldots, s_{k}^{2 n+1}\right]: M \rightarrow \mathbb{C} P^{2 n+1}$.

(3) $s_{k}$ are generic, i.e., there is an uniform $\gamma>0$ such that $\left|\bigwedge^{n} \partial \phi_{k}\right| \geq \gamma$ at all points of $M$. This makes the differential $d \phi_{k}$ injective (moreover it multiplies the length of tangent vectors by an amount bounded below uniformly in $k$ ). This guarantees that the tangent spaces $\left(\phi_{k}\right)_{*} T_{x} M \subset$ $T_{\phi_{k}(x)} \mathbb{C} P^{2 n+1}$ are within distance $O\left(k^{-1 / 2}\right)$ of being complex.

The method for constructing these sequences of embeddings is an extension of that of Donaldson [D1] introduced to obtain asymptotically holomorphic sequences of sections $s_{k}$ of $L^{\otimes k}$ which are transverse to zero in a controlled sense (i.e., there is some $\eta>0$ such that $\left|\partial s_{k}\right|>\eta$ at the points where $\left.\left|s_{k}\right|<\eta\right)$. The sets of zeroes of $s_{k}, W_{k}=Z\left(s_{k}\right)$, are then nearly complex, in the sense that their tangent hyperplanes are within an angle $O\left(k^{-1 / 2}\right)$ of being complex. In particular this gives a construction of symplectic codimension 2 submanifolds.

2000 Mathematics Subject Classification. Primary 53D35.

Key words and phrases. Contact manifold, asymptotically holomorphic, embedding.

Partially supported by The European Contract Human Potential Programme, Research Training Network HPRN-CT-2000-00101. 
Our result follows the streamline of the works of Donaldson [D2] where asymptotically holomorphic sequences of sections $\left(s_{k}^{0}, s_{k}^{1}\right)$ of $\mathbb{C}^{2} \otimes L^{\otimes k}$ are obtained to get a map $M \rightarrow \mathbb{C} P^{1}$ which is a symplectic Lefschetz fibration, and of Auroux $[\mathbf{A u}]$ where asymptotically holomorphic sequences of sections $\left(s_{k}^{0}, s_{k}^{1}, s_{k}^{2}\right)$ of $\mathbb{C}^{3} \otimes L^{\otimes k}$ give ramified asymptotically holomorphic coverings $M \rightarrow \mathbb{C} P^{2}$ for a 4-manifold $M$. In these two cases there are singularities which are shown to be of generic holomorphic type. In our case, in contrast, the main task is the removal of all singularities of the maps from $M$ into the projective space.

Borthwick and Uribe also construct asymptotically holomorphic embeddings into a projective space [BU, theorem 1.2], using a different kind of ideas related to geometric quantization. Their result is stronger since their embeddings are not only asymptotically holomorphic but also asymptotically isometric (although in this case the dimension of the projective space grows with $k$ ).

The asymptotically holomorphic theory has been extended to the case of contact manifolds by Ibort, Martínez and Presas [IMP]. Consider a cooriented contact manifold $(C, \theta)$ of dimension $2 n-1$. Let $L$ be a trivial (complex) line bundle with a connection with curvature form $-i d \theta$. Now fix an almost complex structure $J$ in the symplectic bundle $D=\operatorname{ker} \theta$ which is compatible with the symplectic form $\omega=d \theta$. Let $g_{D}$ be the corresponding metric on $D$ and define the metric $g=g_{D}+\theta \otimes \theta$ on $C$. The operator $\bar{\partial}_{D}$ is defined as the antiholomorphic part of $\nabla$ restricted to $D \subset T C$. Using the metrics $g_{k}=k g$, we have the following definition

Definition 1. A sequence of embeddings $\phi_{k}: C \rightarrow \mathbb{C} P^{2 n+1}$ is asymptotically holomorphic if it satisfies the following conditions:

(1) $d \phi_{k}$ is injective and $\left.d \phi_{k}\right|_{D_{x}}: D_{x} \subset T_{x} C \rightarrow T_{\phi_{k}(x)} \mathbb{C} P^{2 n+1}$ has a left inverse $\theta_{k}$ of norm less than $\gamma^{-1}$ for some small uniform $\gamma>0$, for all $x \in C$.

(2) $\left|\left(\phi_{k}\right)_{*} J-J_{0}\right|_{g_{k}}=O\left(k^{-1 / 2}\right)$ on the subspace $\left(\phi_{k}\right)_{*} D_{x}$, for all $x \in C$.

(3) $\left|\nabla^{p} \phi_{k}\right|_{g_{k}}=O(1)$ and $\left|\nabla^{p-1} \bar{\partial} \phi_{k}\right|=O\left(k^{-1 / 2}\right)$, for all $p \geq 1$.

The result that we want to mention in this note is the following:

THEOREM 2. There is a sequence of asymptotically holomorphic embeddings $\phi_{k}: C \rightarrow \mathbb{C} P^{2 n+1}$ such that the pull-back of the Fubini-Study symplectic form restricted to $D$ is cohomologically trivial.

Denoting by $H$ the hyperplane bundle of $\mathbb{C} P^{2 n+1}$, we actually have that $\phi_{k}^{*} H=$ $L^{\otimes k}\left(\right.$ so $\left.\phi_{k}^{*}\left[\omega_{F S}\right]=[k \omega]\right)$. When the embeddings are asymptotically holomorphic then $\phi_{k}(C)$ are submanifolds of the projective space such that the distribution $\left(\phi_{k}\right)_{*} D \subset T \mathbb{C} P^{2 n+1}$ is within $O\left(k^{-1 / 2}\right)$ of being complex. The proof of theorem 2 follows the lines of the proof of [MPS, theorem 2.11] translated into the context of contact manifolds.

Definition 3. A sequence of sections $s_{k}$ of $\mathbb{C}^{N} \otimes L^{\otimes k}$ is asymptotically contactholomorphic if $\left|\nabla^{p} s_{k}\right|=O(1)$ and $\left|\nabla^{p} \bar{\partial}_{D} s_{k}\right|=O\left(k^{-1 / 2}\right)$, for all $p \geq 0$.

Definition 4. An asymptotically contact-holomorphic sequence of sections $s_{k}$ of $\mathbb{C}^{2 n+2} \otimes L^{\otimes k}$ is $\gamma$-projectizable if $\left|s_{k}\right| \geq \gamma$ at all points of $C$ and for all $k$ large enough.

We start with any asymptotically holomorphic sequence of sections $s_{k}$ and we perturb it as in [MPS, section 2.3] using the following result 
Proposition 5 (Proposition 4.4 in $[\mathbf{P r}]$ ). Let $f: B \times[0,1] \rightarrow \mathbb{C}^{m}$ be a complex valued function, where $B$ is the ball of radius 1 in $\mathbb{C}^{n}$. Let $0<\delta<1 / 2$ and $\sigma=$ $\delta\left(\log \left(\delta^{-1}\right)\right)^{-p}$, where $p$ is a suitable fixed integer depending only on the dimensions $n, m$. Assume that $f_{s}(z)=f(z, s)$ satisfies the following bounds over $B \times[0,1]$,

$$
\left|f_{s}\right| \leq 1,\left|\bar{\partial} f_{s}\right| \leq \sigma,\left|\nabla \bar{\partial} f_{s}\right| \leq \sigma .
$$

Then there exists a smooth curve $w:[0,1] \rightarrow \mathbb{C}^{m}$ such that $|w|<\delta$ and the function $f_{s}-w(s)$ is $\sigma$-transverse to zero over the ball $B(0,1 / 2)$. Moreover, if $\left|\partial f_{s} / \partial s\right|<$ 1 and $\left|\partial \nabla f_{s} / \partial s\right|<1$, we can choose $w$ such that $\left|d^{i} w / d s^{i}\right|<\Phi(\delta), i=1,2$, $d^{j} w / d s^{j}(0)=0$ and $d^{j} w / d s^{j}(1)=0$ for all $j \in \mathbb{N}$, where $\Phi$ is a function depending only on the dimensions $n, m$.

Recall that $f$ is $\sigma$-transverse to zero over $U$ if for every $x \in U$ such that $|f(x)|<\sigma$ we have that $d f$ is surjective and it has a right inverse with norm less than $\sigma^{-1}$. When $m>n$ this condition reduces to $|f(x)| \geq \sigma$ for every $x \in U$. In our case we take an asymptotically holomorphic sequence of sections and perturb it using proposition 5 to get another sequence $s_{k}$ which is $\gamma$-projectizable for some $\gamma>0$. This allows to construct a map $\phi_{k}=\mathbb{P}\left(s_{k}\right): C \rightarrow \mathbb{C} P^{2 n+1}$ which we want to perturb into an immersion. Comparing with the result for symplectic manifolds [MPS, section 2.3] we see that we lose the isotopy result of the constructed sequences of sections.

Definition 6. The sequence $s_{k}$ is $\gamma$-generic if at every point of $C, d \phi_{k}$ is injective and $\left|\bigwedge^{n-1}\left(\partial_{D} \phi_{k}\right)\right| \geq \gamma$ (i.e., $\left.d \phi_{k}\right|_{D}$ multiplies the length of vectors by an amount bounded below uniformly) for all $k$ large enough.

In a way similar to that in the symplectic case [MPS, section 2.4] we may perturb a sequence of sections until we get a $\gamma$-generic one (for some small $\gamma>0$ ) in a process of $n-1$ steps. The main point again is to use the local transversality result in proposition 5. This gives an immersion $\phi_{k}: C \rightarrow \mathbb{C} P^{2 n+1}$ for $k$ large. Finally a very small perturbation in $C^{1}$-norm yields an embedding $\phi_{k}$ without destroying the conditions of projectizability and genericity previously obtained.

The globalization method described in $[\mathbf{I M P}]$ and used to prove theorem 2 produces, starting with any asymptotically holomorphic sequence of sections $s_{k}$ of $\mathbb{C}^{2 n+2} \otimes L^{\otimes k}$, another asymptotically holomorphic sequence $\sigma_{k}=s_{k}+\tau_{k}$ with $\left|\tau_{k}\right|_{C^{0}, g_{k}}<\epsilon$ (for any small $\epsilon>0$ ), such that $\mathbb{P}\left(\sigma_{k}\right)$ is an asymptotically holomorphic sequence of embeddings. We only control the $C^{0}$-norm of $\tau_{k}$, and we may control the $C^{1}$-norm of $\tau_{k}$ in the directions of $D$ (i.e., $\left|\nabla_{D} \tau_{k}\right|$ ), but not the $C^{1}$-norm of $\tau_{k}$ in the Reeb direction. Also we lose the isotopy result of the constructed sequences of sections.

From theorem 2 we obtain the following

Corollary 7 . For $k$ large enough, there are embeddings $\phi_{k}: C \rightarrow \mathbb{C} P^{2 n+1}$ which are weakly dominate, i.e., $\left.\left(\phi_{k}^{*} \omega_{F S}\right)\right|_{D}$ is non-degenerate.

This follows directly from the asymptotic holomorphicity of the map (note that if the map is holomorphic the result is trivially true).

REMARK 8. If $C$ admits a weakly dominate embedding into $\mathbb{C} P^{2 n+1}$ then the bundle $D$ acquires a symplectic structure given by the restriction of the FubiniStudy symplectic form. Therefore $C$ is cooriented. 
Can we perturb the sequence of embeddings to assure that the restriction of the Fubini-Study form to $D$ is exactly $k d \theta$ ? There is no topological constraint for doing this as the restriction of the Fubini-Study form is cohomologous to $k d \theta$. (In [Et] there are examples when this does not happen for a codimension 1 contact manifold $C$ in a symplectic manifold.) To apply Moser's trick in an open neighborhood of the contact manifold, one has to check that the fields defined to apply Moser's trick are complete and this is not clear.

However, if one is able to construct a stronger kind of embedding produced following the ideas of Borthwick and Uribe [BU] one would be able to get this result directly.

Proposition 9. Let $X \subset \mathbb{C} P^{2 n+1}$ be a complex projective submanifold. Then there are asymptotically holomorphic embeddings $\phi_{k}: C \rightarrow \mathbb{C} P^{2 n+1}$ such that $\phi_{k}(C) \bigcap X$ is a contact submanifold of $\phi_{k}(C)$ for $k$ large.

This is a generalization of the ideas contained in [MPS, section 3]. We start with an asymptotically holomorphic embedding $\phi_{k}=\mathbb{P}\left(s_{k}\right)$ as constructed in proposition 2 and we look for another embedding $\hat{\phi}_{k}=\mathbb{P}\left(\sigma_{k}\right)$ with $\left|s_{k}-\sigma_{k}\right|_{C^{0}, g_{k}}<\epsilon$ (for some $\epsilon>0$ ) such that

$$
\angle\left(\left(\hat{\phi}_{k}\right)_{*} D_{x}, T_{\hat{\phi}_{k}(x)} X\right) \geq \eta, \text { for every } x \in C,
$$

for some uniform $\eta>0$. This is achieved as in [MPS, Theorem 3.12] by using proposition 5 . The embedded manifolds $\hat{\phi}_{k}(C) \subset \mathbb{C} P^{2 n+1}$ then satisfy that $\left(\hat{\phi}_{k}\right)_{*} D_{x} \cap T_{\hat{\phi}_{k}(x)} X$ are within distance $O\left(k^{-1 / 2}\right)$ of being complex. Hence $\hat{\phi}_{k}(C) \cap X$ are contact submanifolds of $\hat{\phi}_{k}(C)$.

This construction may provide new examples of contact submanifolds in the spirit of the symplectic examples produced in [MPS]. Also it may be extended to the case when $C$ is not cooriented by taking the double (cooriented) cover $\hat{C}$, embedding it into $\mathbb{C} P^{2 n+1}$ in a $\mathbb{Z}_{2}$-equivariant way (where $\mathbb{Z}_{2}$ acts in $\mathbb{C} P^{2 n+1}$ by the involution $\left.I\left[z_{0}, \ldots, z_{2 n+1}\right]=\left[\bar{z}_{0}, \ldots, \bar{z}_{2 n+1}\right]\right)$, and intersecting with a real subvariety $X \subset \mathbb{C} P^{2 n+1}$ (i.e., an algebraic subvariety invariant by $I$ ).

\section{References}

[Au] D. Auroux, Symplectic 4-manifolds as branched coverings of $\mathbb{C} P^{2}$. Invent. Math. 139 (2000), 551-602.

[BU] D. Borthwick and A. Uribe, Nearly kählerian embeddings of symplectic manifolds. Asian J. Math. 4 (2000), 599-620.

[D1] S. K. Donaldson, Symplectic submanifolds and almost-complex geometry. J. Diff. Geom. 44 (1996), 666-705.

[D2] - Lefschetz pencils on symplectic manifolds. J. Diff. Geom. 53 (1999), 205-236.

[Et] J. Etnyre, Convexity in contact geometry. Topology Appl. 88 (1998), 3-25.

[IMP] A. Ibort, D. Martínez and F. Presas, On the construction of contact submanifolds with prescribed topology. To appear in J. Diff. Geom.

[MPS] V. Muñoz, F. Presas and I. Sols, Almost holomorphic embeddings in grassmannians with applications to singular symplectic submanifolds. math.DG/0002212

[Pr] F. Presas, Lefschetz type pencils on contact manifolds. math.SG/0007034 
Departamento de Matemáticas, Facultad de Ciencias, Universidad Autónoma de MADRID, 28049 MADRID, SPAIN

E-mail address: vicente.munoz@uam.es

Departamento de Álgebra, Facultad de Ciencias Matemáticas, Universidad ComPlutense de Madrid, 28040 Madrid, Spain

E-mail address: fpm@eucmos.sim.ucm.es

Departamento de Álgebra, Facultad de Ciencias Matemáticas, Universidad ComPLUtense De MAdRID, 28040 Madrid, Spain

E-mail address: sols@mat.ucm.es 